\title{
Competitor Identification by Use The Sentiment Classification Based on the User Research
}

\author{
V. Ajay Varma, T. Ramesh Krishna, Mercy Paul Selvan
}

\begin{abstract}
Online shopping's have achieved an immense growth. All like to do it as there is no need to physically to the shop and we have a wide range of collections available in the online sites from which we can actually buy the product. The customers usually tend to purchase a product that has a good customer review and has the highest rating. Numerous reviews are given for a single product and the most of the important reviews are not organized well which makes it disappear from the other reviews. Numerous researchers have worked on structuring the reviews for various purposes. In this work we propose a sentimental analysis of customer reviews for various hotel items. All the items are reviewed by the customers and the proposed work makes an analysis of the reviews obtained for a particular item in all the available shops. This analysis is helpful injudging the most likely consumed food by the customers around and can get to know the competiveness of the product being delivered to the customers. Machine Learning techniques and Natural language Processing (NLP) are used for the proposed work and is observed to produce an efficient result.
\end{abstract}

Keywords-Product Rating, Consumer review, Sentiment Classifier, Natural Language Processing Extraction, Classification,Recognition, Prediction

\section{INTRODUCTION}

Nowadays, usage of Internet has made an outstanding way of expressing the opinions about the products featured online. Numerous websites and applications are present for online purchases. Let it be electronic gadgets, accessories, clothing and be it food everything is being sold out online and we can get it received at our doorsteps. All the products being sold have their own customer reviews. Customer reviews play a vital role in purchasing product. The previous customers who have already purchased the product tend to give a rating to the product purchased and the overall rating of the product changes as per all the reviews obtained for that particular productIn this paper we have proposed a sentimental analysis classifier that is used to classify the reviews and opinions that are obtained for various food items in hotel. Numerous applications are being widely used for delivering food. All the items delivered by a particular hotel has its own customer rating [18]. The proposed work is used to find the rating of a particular food item in all the hotels so that it could be clearly seen that if we want a particular item in which shop it has the highest customer rating. The proposed work uses sentiment analysis classifier to segregate the customer reviews and does an analysis. The

Revised Manuscript Received on July 18, 2019.

V. Ajay Varma, UG Student, Dept. of CSE, Sathyabama University, Chennai, Tamilnadu, India

T. Ramesh Krishna, UG Student, Dept. of CSE, Sathyabama University, Chennai, Tamilnadu, India

Dr. Mercy Paul Selvan, Assistant professor, Dept. of CSE, Sathyabama University, Chennai, Tamilnadu, India performance analysis is done and is been observed that it performs well than the previous existing systems.

\section{RELATED WORK}

There are some researchers are surveyed the online shopping area for reducing the usage problem and so on, in that cases, more system is under consideration for a long. Filtering techniques are also used in the online shopping area [1] - [4]. Reviews for the product in the website will make a product distributor to next level business. More options are provided by the sites which we can purchase the product based on the reviews [5], [6]. Some sites will have language recommendation and some will not have it. There are some area which was surveyed by the researchers, to show up the communication problem between users and the product provider, some reviews are also having good response. Online shopping has some rules in network setup, they will connect the database in the cloud, so that communication will not stuck up at any point of view. There are some methods are her to show the clear idea for the system management. Customized service is very important because while the service was in automation, there will be some errors happen by the system, if it is not then service will go smoothly without any interruption [7] - [11,16]. Domains which are not having the special area for resource then it should have the extra area for the clarifications, there may be the greatest value for the building system [12], [13], some researcher are telling to move the system with automation and customizable service, because if service man is not available in the region or in the line [14], then system will notify them for the intimation of the situation. Then user may wait till situation gets clear. Filtering should be in one area for the observation and system management. Some researchers are telling reviewers to give optional area for their own wordsand satisfaction, fake reviews can be removed by the developer or user in later [15,17], so as we surveyed, there are more points and schemes are pointed for our project.

\section{PROPOSED APPROACH}

In our projected system to spot the aggressiveness between the things, in every things having the amount of options .We use Product Reviews still as building Reviews for Implementation. Of that building Domain Sentiment classification may be extended to convey Service recommendation to users supported their necessities. The 


\section{COMPETITOR IDENTIFICATION BY USE THE SENTIMENT CLASSIFICATION BASED ON THE USER RESEARCH} their meaning. Then we have a tendency to collect the info in unstructured datasets over the multiple domains and apply the Nlp(Natural Language Processing) to spot the similar sorts of reviews on the merchandise.

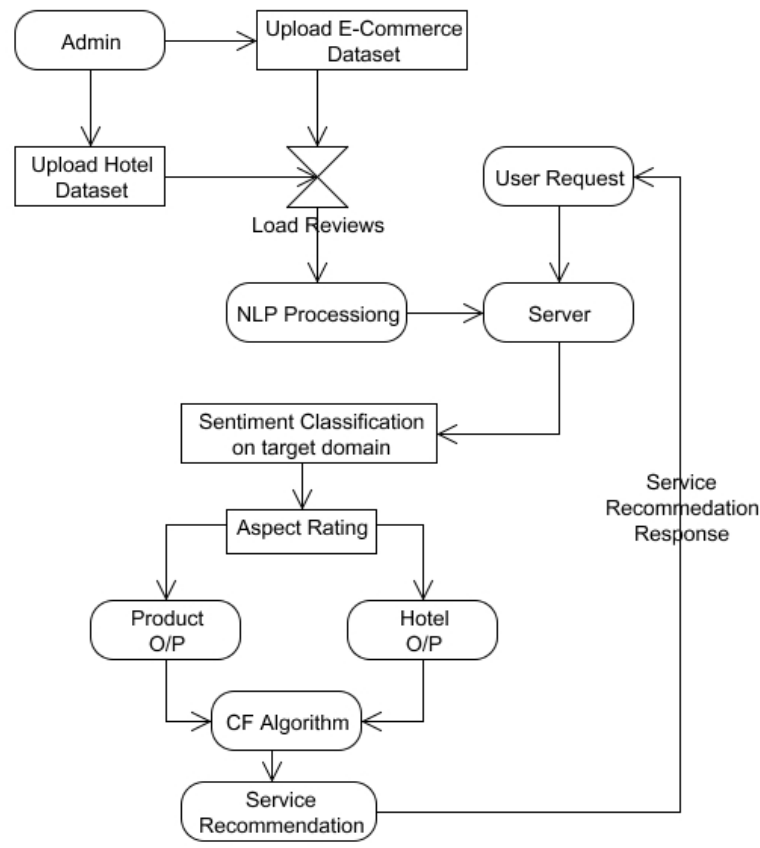

Fig.1. Proposed System

Then apply the cooperative filtering technique for establish the simplest items within the varied domains supported the user reviews and type the things. currently we've the sorted itemslist then we'd like to identity the aggressiveness of the things. therefore we have a tendency to are visiting calculate the aggressiveness of the merchandise, supported the intersection between the similar sorts of product options reviews that are provided by the $n$ variety of user for every product. A user-based CF algorithmic rule is adopted to come up with applicable recommendations. It aims at scheming a customized rating of every candidate service for a user, then presenting a customized service recommendation list shown in Fig.1 and recommending the foremost applicable services to him/her. Then we'd like to seek out the share of aggressiveness between the merchandise that may be calculated supported no of users reviews products/total no of users.

\section{POS Tagging for User Reviews}

Vast assortment of knowledge is retrieved from open supply datasets that are in public obtainable from internet applications like Trip authority and Amazon .The Data's are in CSV or TSV Format. The CSV(Comma separated values) files were scan and manipulated mistreatment Java API that itself developed by US that is developer friendly, light weighted and simply modifiable. The User review for 2 completely different domains were loaded as a CSV or TSV file ,parsed mistreatment api so every review by each client is processed consecutive. The reviews got one by one to POS Tagger that splits every word within the review and tags it supported the elements of Speech the word belongs. user ought to be adding the reviews of the item supported

\section{Constellation the Reviews and side Extraction}

Chucker method is completed on every and each review of all and also the merchandise. The Clunker method can take POS labeled output as input for Grouping the Words supported which means of the Review. Chuncker method is completed in order that we are able to simply extract the sentiment embeddings related to the Aspects of the actual review. The meaty words that ought to be scan unceasingly for correct understanding of the review are marked with punctuation. currently the Aspects in every review are extracted from the POS Tagger result. The Noun and construction Verbs are the key Attributes in any sentence. therefore those things were extracted from the labeled reviews and marked as Aspects of the actual review by a user. currently mappings are done to properly annotate the user review and associated Aspects with the Chunks in it.



Fig.2. Sequence Diagram

3. Building Domain synonym finder not off course Domains

A Domain synonym finder is made reckoning on the Keyword Candidate List and Candidate Services List. Keyword Candidate List is that the and Candidate Services List are mutualist on the Target domains and it is ready before porting the classifier to focus on domain. professional data ought to run for making ready the domain synonym finder. The Domain synonym finder is Updated frequently to urge correct Results of the advice System. currently the Aspects extracted are subjected to domain uncertain supported the target domain.as shown in Sequence diagram Fig.2.

\section{Service Recommendation \& distinguishing Competitions}

The Chunked Reviews of the User is retrieved and also 
the Keywords (Aspects) comparable to the User is analyzed for its Valence and Arousal. Valence means that Weather the Ratings are given for every domain in Target supported the Valence and Arousal for each User of every review. For product reviews the general Rating is currently manipulated by taking average values of every rating of many users of a selected product. In edifice Domain we have a tendency to extend ranking to relinquish customized Service recommendation to user supported needs to user. Ranking is completed for all hotels supported Ratings by similar users mistreatment CF (Collaborative Filtering) and can be sorted based on Bubble kind rule to own the foremost applicable customized Recommendation for the User. Then we want to seek out the share of fight between the merchandise that may be calculated supported no of users reviews products/total no of users.

\section{EXPERIMENTAL RESULTS}

In Fig. 2, we can see the comparison of the existing system and proposed system by choosing the area of response time. It has given good result and achieved the data very well. On deploying the system by checking the data in creating the cloud platform. We have achieved the analysis by checking the system and represented in the graph area. In the graph we can see the number of classes in comparison, in that high level is the proposed method and low level is an existing method. It was compared by seeing the time and achieved well.
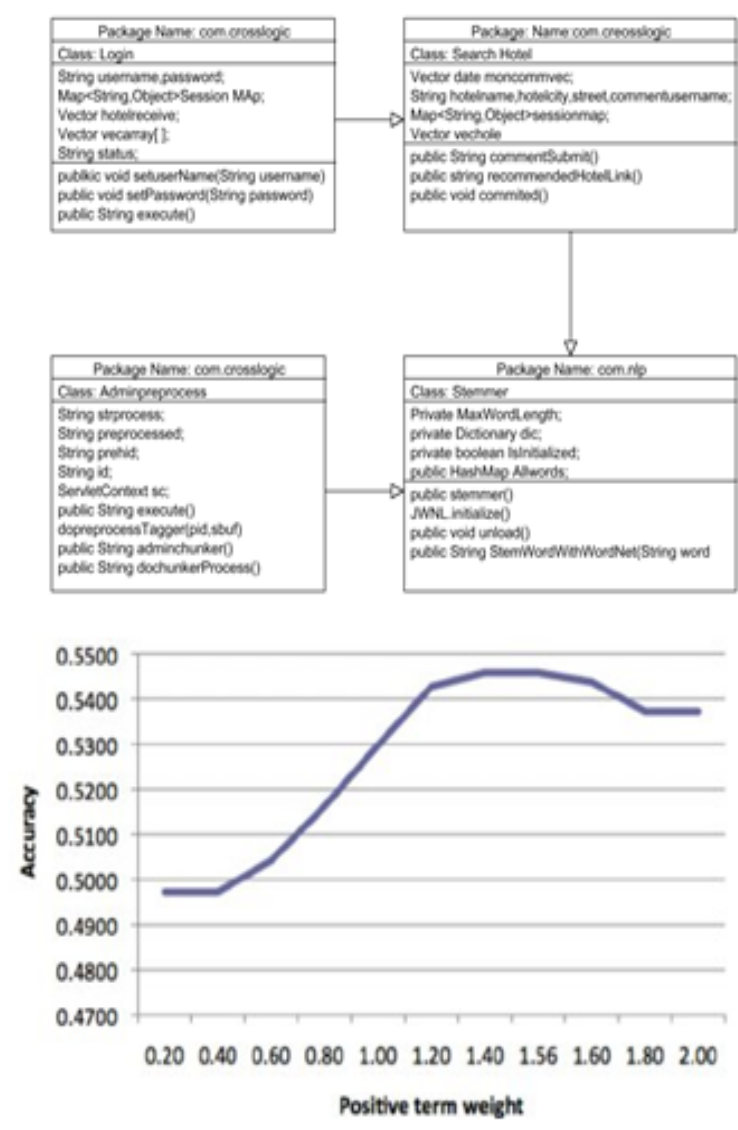

Fig.2. Result Analysis

\section{CONCLUSION}

More usage is occurring in online shopping as same to this, there is a number of attacks are also occurring. To solve these issues and complexity, we have proposed the special scheme for protecting the area which is getting affected by a identification in the shopping. The result was achieved by Broker security with analyzing models. There some comparison shows up well and discussed as per the details proceeded by the researchers. In future work, this method can be used in banking region for securing the user message and banking information.

\section{ACKNOWLEDGEMENT}

We wish to acknowledge the department of science and technology, india and school of computing, sathyabama institute of science and technology, chennai for providing the facilities to do the research under the dst-fist grant project no.sr/fst/eti-364/2014.

\section{REFERENCES}

1. N. Godbole, M. Srinivasaiah, and S. Skiena,(2007),"Large-scale sentiment analysis for news and blogs," in International Conference on Weblogs andSocial Media (ICWSM), pp. 219-222.

2. N. Jakob and I. Gurevych, "Extracting opinion targets ina single- and cross-domain setting with conditionalrandom fields," presented at the Proceedings of the 2010Conference on Empirical Methods in Natural LanguageProcessing, Cambridge, Massachusetts

3. Jingbo Zhu, Huizhen Wang, Muhua Zhu," Aspect-Based Opinion Polling from CustomerReviews," IEEE transactions on Affective Computing, vol. 2, no. 1,January 2011,pp.37-49.

4. Kamala, V. R., and L. MaryGladence. "An optimal approach for social data analysis in Big Data." In 2015 International Conference on Computation of Power, Energy, Information and Communication (ICCPEIC), pp 0205-0208. IEEE, 2015

5. Kang Liu, LihengXu and Jun Zhao," CoextractingOpinion Targets and Opinion Words from OnlineReviews Based on the world alignement model.

6. Bing Liu, "Sentiment Analysis and Opinion Mining,Morgan \& Claypool Publishers,"2012,pp.7-140.

7. Jingbo Zhu, Huizhen Wang, Muhua Zhu," AspectBasedOpinion Polling from Customer Reviews," IEEEtransactions on Affective Computing, vol. 2, no.1,January 2011,pp.37-49

8. Jingbo Zhu, Huizhen Wang, Muhua Zhu," Aspect-Based Opinion Polling from Customer Reviews," IEEE transactions on Affective Computing, vol. 2, no. 1,January 2011,pp.37-49.

9. Kang Liu, LihengXu and Jun Zhao," Co-extracting Opinion Targets and Opinion Words from Online Reviews Based on the Word Alignment Model ,"Journal of Latex class file, Vol. 6, no. 1, January 2007,pp.1-14.

10. Mani, I., Maybury, M.T. (eds.)," Advances in Automatic Text Summarization,".MIT Press (1999).

11. Ohana.B., Tierney.B, "Sentiment classification of reviews using SentiWordNet," 9th. IT\&T Conference, Dublin Institute ofTechnology, Dublin, Ireland, 22nd.23rd. October, 2009.

12. B.Pang,L.Lee,andS.Vaithyanathan," Thumbs up? 
Sentiment classification using machine learning techniques,"inProc EMNLP, Philadelphia, PA, USA, 2002, pp. 79-86.

13. Richa Sharma, Shweta Nigam, Rekha Jain," Polarity detection at sentence level,". International journal of computer applications(0975 - 8887), Volume 86 - No 11.

14. Yang, Y," An evaluation of statistical approaches to text categorization. Journal of Information Retrieval ,Vol.1,Issue(1-2),1999pp. 69-90.

YuanbinWu, Qi Zhang, Xuanjing Huang, LideWu ," Phrase Dependency Parsing for Opinion Mining," Proceedings of the 2009 Conference on Empirical Methods in Natural Language Processing, pp.15331541.

15. Gladence, L. Mary, M. Karthi, and V. Maria Anu. "A statistical comparison of logistic regression and different Bayes classification methods for machine learning." ARPN Journal of Engineering and Applied Sciences 10, no. 14 (2015): 5947-5953.

16. Anu, V. Maria, GS Anandha Mala, and K. Mathi. "Comparison of RFID data processing using dimensionality reduction techniques." In 2014 International Conference on Control, Instrumentation, Communication andComputational Technologies (ICCICCT), pp. 265-268. IEEE, 2014.

17. Dr.R.Subhashini and Akila G, "Valence arousal similarity based recommendation services ", IEEE International Conference on Circuit, Power and Computing Technologies, ICCPCT 2015.

18. Dr J.Jabez, "User based efficient video recommendation system", International conference on intelligent data communication technologies and Internet of Things (ICICI 2018). 